\title{
ADVANCED QUANTUM MECHANICS
}


This page intentionally left blank 
ADVANCED QUANTUM

MIECEIANICS

\section{FREEMAN DYSON}

Institute for Advenced Stualy, Princeton, USA

TRANSCRIBED BY

DAVID DERBES

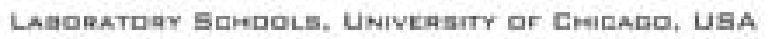




\title{
Published by
}

World Scientific Publishing Co. Pte. Ltd.

5 Toh Tuck Link, Singapore 596224

USA office: 27 Warren Street, Suite 401-402, Hackensack, NJ 07601

UK office: 57 Shelton Street, Covent Garden, London WC2H 9HE

\section{British Library Cataloguing-in-Publication Data}

A catalogue record for this book is available from the British Library.

\begin{abstract}
ADVANCED QUANTUM MECHANICS
Copyright (C) 2007 by World Scientific Publishing Co. Pte. Ltd.

All rights reserved. This book, or parts thereof, may not be reproduced in any form or by any means, electronic or mechanical, including photocopying, recording or any information storage and retrieval system now known or to be invented, without written permission from the Publisher.
\end{abstract}

For photocopying of material in this volume, please pay a copying fee through the Copyright Clearance Center, Inc., 222 Rosewood Drive, Danvers, MA 01923, USA. In this case permission to photocopy is not required from the publisher.

ISBN-13 978-981-270-622-5

ISBN-10 981-270-622-4

ISBN-13 978-981-270-661-4 (pbk)

ISBN-10 981-270-661-5 (pbk) 


\section{Preface}

Both Kaiser's admirable Drawing Theories Apart [8] and Schweber's masterful QED and the Men Who Made It [7] refer frequently to the famous lectures on quantum electrodynamics given by Freeman Dyson at Cornell University in 1951. Two generations ago, graduate students (and their professors) wishing to learn the new techniques of QED passed around copies of Dyson's Cornell lecture notes, then the best and fullest treatment available. Textbooks appeared a few years later, e.g. by Jauch \& Rohrlich [25] and Schweber [6], but interest in Dyson's notes has never fallen to zero. Here is what the noted theorist E. T. Jaynes wrote in an unpublished article [26] on Dyson's autobiographical Disturbing the Universe, 1984:

But Dyson's 1951 Cornell course notes on Quantum Electrodynamics were the original basis of the teaching I have done since. For a generation of physicists they were the happy medium: clearer and better motivated than Feynman, and getting to the point faster than Schwinger. All the textbooks that have appeared since have not made them obsolete. Of course, this is to be expected since Dyson is probably, to this day, best known among the physicists as the man who first explained the unity of the Schwinger and Feynman approaches.

As a graduate student in Nicholas Kemmer's department of theoretical physics (Edinburgh, Scotland) I had heard vaguely about Dyson's lectures (either from Kemmer or from my advisor, Peter Higgs) and had read his classic papers [27], [28] in Schwinger's collection [4]. It never occurred to me to ask Kemmer for a copy of Dyson's lectures which he almost certainly had. 
My interest in the legendary notes was revived thirty years later by the Kaiser and Schweber books. Within a few minutes Google led to scans of the notes [29] at the Dibner Archive (History of Recent Science \& Technology) at MIT, maintained by Karl Hall, a historian at the Central European University in Budapest, Hungary. He had gotten permission from Dyson to post scanned images of the Cornell notes. Through the efforts of Hall, Schweber and Babak Ashrafi these were uploaded to the Dibner Archive. To obtain a paper copy would require downloading almost two hundred images, expensive in time and storage. Was there a text version? Had anyone retyped the notes? Hall did not know, nor did further searching turn anything up. I volunteered to do the job. Hall thought this a worthwhile project, as did Dyson, who sent me a copy of the second edition, edited by Michael J. Moravcsik. (This copy had originally belonged to Sam Schweber.) Dyson suggested that the second edition be retyped, not the first. Nearly all of the differences between the two editions are Moravcsik's glosses on many calculations; there is essentially no difference in text, and (modulo typos) all the labeled equations are identical.

Between this typed version and Moravcsik's second edition there are few differences; all are described in the added notes. (I have also added references and an index.) About half are corrections of typographical errors. Missing words or sentences have been restored by comparison with the first edition; very infrequently a word or phrase has been deleted. A few changes have been made in notation. Intermediate steps in two calculations have been corrected but change nothing. Some notes point to articles or books. No doubt new errors have been introduced. Corrections will be welcomed! The young physicists will want familiar terms and notation, occasionally changed from 1951; the historians want no alterations. It was not easy to find the middle ground.

I scarcely knew $\mathrm{AT}_{\mathrm{EX}}$ before beginning this project. My friend (and Princeton '74 classmate) Robert Jantzen was enormously helpful, very generous with his time and his extensive knowledge of IATEX. Thanks, Bob. Thanks, too, to Richard Koch, Gerben Wierda and their colleagues, who have made IATEX so easy on a Macintosh. George Grätzer's textbook Math into ${ }^{A} T_{E} X$ was never far from the keyboard. No one who types technical material should be ignorant of LATEX.

This project would never have been undertaken without the approval of Prof. Dyson and the efforts of Profs. Hall, Schweber and Ashrafi, who made the notes accessible. I thank Prof. Hall for his steady encouragement 
through the many hours of typing. I thank Prof. Dyson both for friendly assistance and for allowing his wonderful lectures to become easier to obtain, to be read with pleasure and with profit for many years to come.

Originally, the typed version was meant to serve as an adjunct to Karl Hall's scanned images at the Dibner site. Bob Jantzen, a relativist active in research, insisted that it also go up at the electronic physics preprint site arXiv.org, and after a substantial amount of work by him, this was arranged. A few weeks later the alert and hardworking team at World Scientific ${ }^{1}$ got in touch with Prof. Dyson, to ask if he would allow them to publish his notes. He was agreeable, but told them to talk to me. I was delighted, but did not see how I could in good conscience profit from Prof. Dyson's work, and suggested that my share be donated to the New Orleans Public Library, now struggling to reopen after the disaster of Hurricane Katrina. Prof. Dyson agreed at once to this proposal. I am very grateful to him for his contribution to the restoration of my home town.

\author{
David Derbes \\ Laboratory Schools \\ University of Chicago \\ loki@uchicago.edu \\ 11 July 2006
}

\footnotetext{
${ }^{1}$ World Scientific is very grateful to Professor Freeman Dyson and Dr David Derbes for this magnificent manuscript.
} 


\section{Contents}

Preface $\quad$ v

Generally used Notation xiii

1 Introduction $\quad 1$

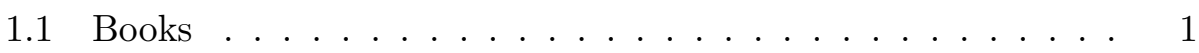

1.2 Subject Matter . . . . . . . . . . . . . . . . 1

1.3 Detailed Program . . . . . . . . . . . . . . . . 2

1.4 One-Particle Theories . . . . . . . . . . . . . 3

2 The Dirac Theory 5

2.1 The Form of the Dirac Equation . . . . . . . . 5

2.2 Lorentz Invariance of the Dirac Equation . . . . . . . . . 7

2.3 To Find the $\mathrm{S} \ldots \ldots \ldots \ldots \ldots$

2.4 The Covariant Notation . . . . . . . . . . . . . 11

2.5 Conservation Laws. Existence of Spin . . . . . . . . . . . . . . 12

2.6 Elementary Solutions . . . . . . . . . . . . . . . . . 13

2.7 The Hole Theory . . . . . . . . . . . . . . . . . . . . . . 14

2.8 Positron States . . . . . . . . . . . . . . . . . 15

2.9 Electromagnetic Properties of the Electron . . . . . . . . 16

2.10 The Hydrogen Atom . . . . . . . . . . . . . . . . 18

2.11 Solution of Radial Equation . . . . . . . . . . 20

2.12 Behaviour of an Electron in a Non-Relativistic Approximation .................... 23

2.13 Summary of Matrices in the Dirac Theory in Our Notation . . . . . . . . . . . . . 26 
2.14 Summary of Matrices in the Dirac Theory in the Feynman Notation . . . . . . . . . . . . . 28

3 Scattering Problems and Born Approximation 31

3.1 General Discussion . . . . . . . . . . . . . . . . 31

3.2 Projection Operators . . . . . . . . . . . . . . . . . 32

3.3 Calculation of Traces . . . . . . . . . . . . . . . . . 34

3.4 Scattering of Two Electrons in Born Approximation.

The Møller Formula . . . . . . . . . . . . . . . . . . . . . . 39

3.5 Relation of Cross-sections to Transition Amplitudes . . . . . 41

3.6 Results for Møller Scattering . . . . . . . . . . . . . . 43

3.7 Note on the Treatment of Exchange Effects . . . . . . . . . 44

3.8 Relativistic Treatment of Several Particles . . . . . . . . . . 45

4 Field Theory $\quad 47$

4.1 Classical Relativistic Field Theory . . . . . . . . . . . . 47

4.2 Quantum Relativistic Field Theory . . . . . . . . . . . . . . 51

4.3 The Feynman Method of Quantization . . . . . . . . . . . 52

4.4 The Schwinger Action Principle . . . . . . . . . . . 53

4.4.1 The Field Equations . . . . . . . . . . . 55

4.4.2 The Schrödinger Equation for the State-function . . . 55

4.4.3 Operator Form of the Schwinger Principle . . . . . . 56

4.4.4 The Canonical Commutation Laws . . . . . . . . . 57

4.4.5 The Heisenberg Equation of Motion for the Operators . . . . . . . . . . . . 58

4.4.6 General Covariant Commutation Laws . . . . . . . . . 58

4.4 .7 Anticommuting Fields . . . . . . . . . . . 59

5 Examples of Quantized Field Theories $\quad 61$

5.1 The Maxwell Field . . . . . . . . . . . . . . . . . . . . 61

5.1.1 Momentum Representations . . . . . . . . . . . . 63

5.1 .2 Fourier Analysis of Operators . . . . . . . . . . . 65

5.1.3 Emission and Absorption Operators . . . . . . . . 65

5.1 .4 Gauge-Invariance of the Theory . . . . . . . . . . . 67

5.1 .5 The Vacuum State . . . . . . . . . . . . . . . . 68

5.1.6 The Gupta-Bleuler Method . . . . . . . . . . . 70

5.1.7 Example: Spontaneous Emission of Radiation . . . . . 71

5.1 .8 The Hamiltonian Operator . . . . . . . . . . . . 74

5.1 .9 Fluctuations of the Fields . . . . . . . . . . 75 
5.1.10 Fluctuation of Position of an Electron in a Quantized Electromagnetic Field. The Lamb Shift . . . . . . . 77

5.2 Theory of Line Shift and Line Width . . . . . . . . . . 79

5.2 .1 The Interaction Representation . . . . . . . . . . . 80

5.2.2 The Application of the Interaction Representation to the Theory of Line-Shift and Line-Width . . . . . . 82

5.2.3 Calculation of Line-Shift, Non-Relativistic Theory . . 87

5.2.4 The Idea of Mass Renormalization . . . . . . . . . . 88

5.3 Field Theory of the Dirac Electron, Without Interaction . . . 91

5.3.1 Covariant Commutation Rules . . . . . . . . . . . 92

5.3.2 Momentum Representations . . . . . . . . . . . . . . 94

5.3.3 Fourier Analysis of Operators . . . . . . . . . . . . 94

5.3.4 Emission and Absorption Operators . . . . . . . . 95

5.3.5 Charge-Symmetrical Representation . . . . . . . . 96

5.3 .6 The Hamiltonian . . . . . . . . . . . . . . . . . 97

5.3.7 Failure of Theory with Commuting Fields . . . . . . . 98

5.3.8 The Exclusion Principle . . . . . . . . . . . . . . . . 98

5.3 .9 The Vacuum State . . . . . . . . . . . . . . . . . . 99

5.4 Field Theory of Dirac Electron in External Field . . . . . . . 100

5.4.1 Covariant Commutation Rules . . . . . . . . . . . 101

5.4 .2 The Hamiltonian . . . . . . . . . . . . . . . . . . . 104

5.4.3 Antisymmetry of the States . . . . . . . . . . . 105

5.4.4 Polarization of the Vacuum . . . . . . . . . . . 106

5.4.5 Calculation of Momentum Integrals . . . . . . . . . 111

5.4.6 Physical Meaning of the Vacuum Polarization . . . . . 115

5.4.7 Vacuum Polarization for Slowly Varying

Weak Fields. The Uehling Effect . . . . . . . . . . . . 119

5.5 Field Theory of Dirac and Maxwell Fields in Interaction . . . . . . . . . . . . . . . 120

5.5.1 The Complete Relativistic Quantum Electrodynamics . . . . . . . . . . . 120

5.5.2 Free Interaction Representation . . . . . . . . . . . . 122

6 Free Particle Scattering Problems $\quad 125$

6.1 Møller Scattering of Two Electrons . . . . . . . . . . . . 126

6.1.1 Properties of the $D_{F}$ Function $\ldots \ldots \ldots$. . . . . 128

6.1.2 The Møller Formula, Conclusion . . . . . . . . . . . 129

6.1.3 Electron-Positron Scattering . . . . . . . . . . 130 
6.2 Scattering of a Photon by an Electron. The Compton Effect. Klein-Nishina Formula . . . . . . . . . . . . . . . . . 130

6.2.1 Calculation of the Cross-Section . . . . . . . . . 133

6.2 .2 Sum Over Spins . . . . . . . . . . . . . . . . 134

6.3 Two Quantum Pair Annihilation . . . . . . . . . . . . 139

6.4 Bremsstrahlung and Pair Creation in the Coulomb Field of an Atom . . . . . . . . . . . . . . . . . . . 142

7 General Theory of Free Particle Scattering 145

7.1 The Reduction of an Operator to Normal Form . . . . . . . . 148

7.2 Feynman Graphs . . . . . . . . . . . . . . . . . . 152

7.3 Feynman Rules of Calculation . . . . . . . . . . . . . 155

7.4 The Self-Energy of the Electron . . . . . . . . . . . . . 158

7.5 Second-Order Radiative Corrections to Scattering . . . . . . . 162

7.6 The Treatment of Low-Frequency Photons. The Infra-Red Catastrophe . . . . . . . . . . . . . . . 181

8 Scattering by a Static Potential. Comparison with Experimental Results $\quad 183$

8.1 The Magnetic Moment of the Electron . . . . . . . . . . . . 189

8.2 Relativistic Calculation of the Lamb Shift . . . . . . . . . . . 191

8.2.1 Covariant Part of the Calculation . . . . . . . . . . . 193

Covariant Part of the Calculation . . . . . . . . . . . . . 193

8.2.2 Discussion and the Nature of the $\Phi$-Representation . . 196

8.2.3 Concluding Non-Covariant Part of the Calculation . . 198

8.2.4 Accuracy of the Lamb Shift Calculation . . . . . . . 202

$\begin{array}{ll}\text { Notes } & 205\end{array}$

$\begin{array}{ll}\text { References } & 210\end{array}$

$\begin{array}{ll}\text { Index } & 215\end{array}$ 


\section{Generally Used Notation}

$$
\begin{aligned}
A^{*}= & \text { complex conjugate transposed (Hermitian conjugate) } \\
A^{+}= & \text {complex conjugate (not transposed) } \\
\bar{A}= & A^{*} \beta=A^{*} \gamma_{4}=\text { adjoint } \\
A^{-1}= & \text { inverse } \\
A^{\mathrm{T}}= & \text { transposed } \\
\mathbb{I}= & \text { identity matrix or operator } \\
\operatorname{Tr} A= & \text { trace of matrix } A \text { (sum of all diagonal elements) } \\
\not & =\sum_{\mu} a_{\mu} \gamma_{\mu} \text { (This slash notation is also colorfully known as the } \\
& \text { Feynman dagger.) }
\end{aligned}
$$

